\title{
Mortalidade por suicídio: realidade de uma cidade no interior do nordeste brasileiro
}

\section{Suicide mortality: the reality of a city in the interior of the Northeast of Brazil \\ Mortalidad por suicidio: realidad de un pueblo del noreste de Brasil}

\author{
Sérgio André de Souza Júnior (iD \\ Universidade de Fortaleza - Fortaleza (CE) - Brasil \\ Cássia Ferreira Rodrigues i \\ Universidade Federal do Ceará - Fortaleza (CE) - Brasil
}

\begin{abstract}
RESUMO
Objetivo: Delinear as características epidemiológicas da mortalidade por suicídio em uma cidade no interior do Nordeste brasileiro. Métodos: Trata-se de um estudo descritivo e retrospectivo realizado com dados secundários. Coletaram-se dados referentes aos 67 casos de suicídio ocorridos em uma cidade no interior do Ceará, extraídos do Sistema de Informações sobre Mortalidade do Ministério da Saúde, de 2000 a 2015. O estudo incluiu mortes por lesões autoprovocadas intencionalmente, de acordo com

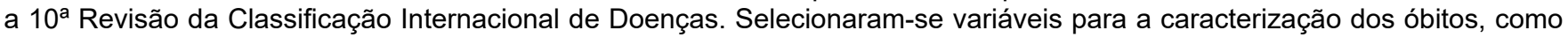
gênero, faixa etária, estado civil, raça/cor, ocupação e método utilizado. Resultados: Estimou-se o coeficiente de mortalidade específica em 8,77/100 mil habitantes no período avaliado. A maior frequência encontrada foi na população masculina (76,1\%), de 40 a 49 anos (32,8\%), com estado civil solteiro (53\%), de raça/cor parda $(68,4 \%)$ e com atuação laboral agrícola (64,2\%). Verificou-se coeficiente preocupante entre idosos na faixa etária entre 60-69 anos (12,44/100mil habitantes). Enforcamento e autointoxicação por pesticidas configuram-se como os dois principais meios utilizados. Conclusão: Os dados de mortalidade por suicídio no município encontram-se em consonância com as estatísticas globais, mas acima das taxas estaduais e nacionais de suicídio. O perfil das pessoas que cometeram suicídio é composto predominantemente por homens, etnia parda, solteiros e que exercem atividade agrícola como ocupação.
\end{abstract}

Descritores: Suicídio; Aplicações da Epidemiologia; Causas Externas.

\section{ABSTRACT}

Objective: To describe the epidemiological characteristics of suicide mortality in a city in the interior of the Northeast of Brazil. Methods: This is a descriptive and retrospective study conducted with secondary data. Data were collected on the 67 cases of suicide, which occurred in a city in the interior of the Northeast, extracted from the Mortality Information System of the Ministry of Health, from 2000 to 2015. The study included deaths from Intentionally Self-Injured Injuries according to the 10th Revision of the International Classification of Diseases. Variables were selected to characterize death, such as gender, age group, marital status, race/color, occupation, and method used. Results: The specific mortality coefficient estimative was at $8.77 / 100$ thousand inhabitants in the evaluated period. The highest frequency was in the male population (76.1\%), 40 to 49 years old (32.8\%), with single marital status (53\%), race / brown color (68.4\%), with work experience agricultural (64.2\%). There was a worrying coefficient among the elderly aged 60-69 years (12.44/100 thousand inhabitants. Hanging and self-poisoning by pesticides are two main means used. Conclusion: The suicide mortality data in the municipality are in line with global statistics and above state and national suicide rates. The profile of people who committed suicide is predominantly composed of men, mixed ethnicity, single, and who exercise agricultural activity as an occupation.

Descriptors: Suicide; Uses of Epidemiology; External Causes.

\section{RESUMEN}

Objetivo: Delinear las características epidemiológicas de mortalidad por suicidio de un pueblo del Noreste de Brasil. Métodos: Se trata de un estudio descriptivo y retrospectivo realizado con datos secundarios. Se ha recogido los datos de 67 casos de suicidio ocurridos en un pueblo de Ceará registrado en el Sistema de Informaciones de Mortalidad del Ministerio de la Salud 
entre 2000 y 2015. El estudio ha incluido las muertes por lesiones auto provocadas a propósito según la $10^{a}$ Revisión de la Clasificación Internacional de Enfermedades. Se ha elegido variables para la caracterización de los óbitos como el género, la franja de edad, el estado civil, la raza/el color de la piel, la ocupación y el método utilizado. Resultados: Se ha estimado el coeficiente de mortalidad especifico de 8,77/100 mil habitantes para el período evaluado. La mayor frecuencia encontrada ha sido de la población masculina (76,1\%) entre 40 y 49 años (32,8\%), solteros (53\%), de raza/color de la piel pardo (68,4\%) y con actuación laboral agrícola (64,2\%). Se ha verificado el coeficiente preocupante entre mayores en la franja de edad entre $60 \mathrm{y}$ 69 años (12,44/100 mil habitantes). Ahorcamiento y auto intoxicación por pesticidas son los dos principales medios utilizados. Conclusión: Los datos de mortalidad por suicidio del municipio están en consonancia con las estadísticas globales pero por encima de las tasas estaduales y nacionales de suicidio. El perfil de las personas que han practicado el suicidio está formado de hombres, con la etnia parda, solteros y que tienen la ocupación en la actividad agrícola.

Descriptores: Suicidio; Usos de la Epidemiología; Causas Externas.

\section{INTRODUÇÃO}

O comportamento suicida é um fenômeno complexo e multifatorial que engloba formas de violência autoinfligidas, em que o indivíduo intencionalmente tira a própria vida ${ }^{(1-3)}$. O suicídio abrange atitudes que variam desde atos de autoagressão até ações mais graves, como ideação de morte, elaboração de plano e a obtenção dos meios para a realização do ato ${ }^{(4)}$. Apresenta-se como grave problema de saúde pública, já que é mencionado entre as dez principais causas de morte em todos espectros etários e abrange todas as regiões do mundo ${ }^{(1-3)}$.

O comportamento suicida é causado, geralmente, por transtornos psiquiátricos, estresse, conflitos interpessoais, violência e impulsividade ${ }^{(4-7)}$. Entre os transtornos relacionados com o risco de suicídio, destacam-se: os transtornos do humor, sobretudo a depressão e transtorno bipolar; os transtornos mentais e de comportamento, decorrentes do uso de substâncias psicoativas, como o alcoolismo e tabagismo; e os transtornos de personalidade e a esquizofrenia ${ }^{(2,4,8,9)}$. O enfrentamento do etilismo e do tabagismo são temas prioritários da Política Nacional de Promoção de Saúde ${ }^{(10)}$.

O delineamento do perfil epidemiológico do suicídio no Brasil apresenta crescimento significativo nas últimas décadas. Em comparação com outros países, o coeficiente de mortalidade por suicídio é menor, porém, quando considerados os números absolutos, o país ocupa a oitava posição mundial e evolui, conforme estudos de séries temporais, com tendência de crescimento progressivo ${ }^{(6,9,11)}$.

Os coeficientes de mortalidade são estimados em torno de 10.000 óbitos anuais, o que equivale a 5,5 óbitos/100 mil habitantes em 2015 $5^{(2,9,12)}$. Um estudo ecológico sobre suicídio no Brasil apontou altos coeficientes nos estados do Sul, com destaque para o Rio Grande do Sul. Entre os municípios brasileiros, os coeficientes mais elevados ocorreram em Taipas do Tocantins, no estado do Tocantins (79,68 óbitos/100 mil habitantes), em Itaporã, no Mato Grosso do Sul (75,15 óbitos/100 mil habitantes), e em Mampituba, Rio Grande do Sul (52,98 óbitos/100 mil habitantes) ${ }^{(13)}$.

Devido à grande repercussão do fenômeno no contexto mundial, a prevenção ao suicídio tornou-se pilar relevante para promoção da saúde e inseriu-se no Plano de Ação em Saúde Mental da Organização Mundial de Saúde, cujo objetivo é reduzir as taxas de suicídios nos países em $10 \%$ até o ano de $2020^{(14,15)}$. No Brasil, o Ministério da Saúde (MS) promove a Estratégia Nacional para Prevenção do Suicídio desde $2006^{(16)}$. A ampliação da cobertura do serviço de saúde mental, o aumento da acessibilidade aos Centros de Atenção Psicossocial (CAPS), a implementação de iniciativas educacionais comunitárias e as terapias integrativas são exemplos de ações e medidas propostas em âmbito nacional ${ }^{(5,13,16)}$.

Em abril de 2019, foi sancionada a Lei n. ${ }^{\circ} 13.819$, que institui a Política Nacional de Prevenção da Automutilação e do Suicídio. A lei cria um sistema nacional, em cooperação com estados, municípios e o Distrito Federal, para prevenção do suicídio e da automutilação. Essa medida constitui um marco legal para que possíveis regulamentações possam ser implementadas no território nacional a fim de prevenir o suicídio ${ }^{(17)}$.

Essas ações surgem como iniciativas do MS para tentar diminuir a incidência de casos no Brasil, visando à redução das taxas de mortes por suicídio e os danos causados às pessoas envolvidas direta e indiretamente nesse ato. Tais políticas são importantes, uma vez que a rede de saúde enfrenta desafios para desenvolver ações preventivas e assistenciais relacionadas ao suicídio(13,16,17).

Assim, visando a melhoria da promoção de saúde mental e a elaboração de subsídios para o planejamento de ações preventivas nos serviços de saúde, este estudo apresenta como objetivo delinear as características epidemiológicas da mortalidade por suicídio em uma cidade no interior do Nordeste brasileiro. 


\section{MÉTODOS}

Trata-se de estudo descritivo, retrospectivo e de série temporal, de 2000 a 2015, realizado com dados secundários. Selecionaram-se dados provenientes do Sistema de Informações sobre Mortalidade (SIM) sobre casos de suicídio ocorridos no município de Morada Nova, cidade do interior do estado do Ceará, com distância estimada em 162 quilômetros da capital Fortaleza, localizada na Mesorregião do Jaguaribe e microrregião do Baixo Jaguaribe. Possui população avaliada em 61.890 pessoas, segundo estimativas populacionais do ano de 2019 , e área territorial de $2.778,578$ quilômetros quadrados e densidade demográfica avaliada em 22,33 hab./km²(18,19).

O critério de inclusão das causas de óbito referentes ao suicídio seguiu a categorização da décima versão da Classificação Estatística Internacional de Doenças e Problemas Relacionados à Saúde (CID-10) ${ }^{(20)}$, selecionadas pelos agrupamentos que englobam as lesões autoprovocadas intencionalmente: medicamentos, substâncias biológicas e não especificadas (X60-X64); pesticidas e produtos químicos (X68-X69); enforcamento, estrangulamento e sufocação (X70); fumaça, pelo fogo e por chamas (X75-X77); arma branca e objetos contundentes (X78-X79) e meios não especificados (X84).

Selecionaram-se variáveis sociodemográficas para a caracterização dos óbitos, como gênero, faixa etária (10 a 19, 20 a 29, 30 a 39, 40 a 49, 50 a 59, 60 a 69, 70 a 79 anos), estado civil, raça/cor e ocupação laboral.

Definiu-se a quantificação da população residente por meio de estimativas populacionais, dados do Censo e projeções intercensitárias do Instituto Brasileiro de Geografia e Estatística (IBGE) ${ }^{(18,19)}$. As projeções de população para o nível geográfico municipal por faixa etária, utilizadas para o cálculo de coeficiente de mortalidade específica, apresentam disponibilidade até o ano de 2015. Por esse motivo, selecionou-se o período de estudo entre 2000 e 2015.

Calculou-se o coeficiente de mortalidade específica por suicídio pela soma do número de óbitos no período de 2000 a 2015, como numerador multiplicado por 100 mil habitantes, e no denominador considerou-se o somatório da população residente no mesmo período registrado ${ }^{(21)}$.

Utilizou-se o programa Statistical Package for Social Sciences (SPSS Inc.), versão 23.0, para tabulação, distribuição e análise estatística dos dados.

Este estudo atendeu aos preceitos éticos do Conselho Nacional de Saúde (CNS), Resolução n. ${ }^{\circ} 510$, de 7 de abril de 2016. Devido a utilização exclusiva dos dados de domínio público, disponibilizados nos sítios eletrônicos do Departamento de Informática do Sistema Único de Saúde (DATASUS) e sem identificação dos sujeitos, é dispensada a submissão ao Comitê de Ética em Pesquisa (CEP).

\section{RESULTADOS}

No período de 2000 a 2015, registram-se 67 óbitos por suicídio na cidade de Morada Nova, com coeficiente de mortalidade de 8,77/100 mil habitantes. O coeficiente mínimo indicou 3,92/100 mil habitantes em 2009, e o máximo ocorreu em 2005, com estimativa de 17,8 óbitos/100 mil habitantes, conforme Figura 1.

Durante o intervalo temporal do estudo (2000-2015), não houve registro de óbito apenas em 2014. O coeficiente de mortalidade por suicídio no sexo masculino alcançou 13,21 óbitos/100 mil habitantes, sendo superior ao feminino, que correspondeu a 4,25 óbitos/100 mil habitantes, isto é, equivalente a uma razão de 3,18:1. Os coeficientes de mortalidade por suicídio em mulheres superaram as taxas masculinas apenas em 2002 e em 2004.

$\mathrm{Na}$ Tabela I, analisando a proporção de óbitos segundo características sociodemográficas, observa-se que o suicídio abrangeu majoritariamente a população masculina $(76,1 \%)$. A faixa etária prevaleceu entre 40 a 49 anos de idade $(32,8 \%$ ) e o estado civil solteiro(a) predominou, com um total de $52,2 \%$ dos casos, seguido pelo estado civil casado(a), com $37,3 \%$. A raça parda apresentou prevalência em $58,2 \%$ dos casos. Em relação à situação ocupacional, houve predomínio de $64,2 \%$ de trabalhadores do setor agropecuário, seguidos por trabalhadores em serviços de reparação e manutenção (10,4\%).

Em relação à faixa etária, os maiores coeficientes de mortalidade por suicídio são ordenados entre 40-49 anos (20,57/100 mil habitantes) e 60-69 anos (12,44/100 mil habitantes), como demonstrado na Tabela II.

Quanto aos métodos utilizados, 49,3\% ( $n=33)$ dos óbitos decorreram de lesões autoprovocadas intencionalmente por enforcamento, estrangulamento e sufocação, seguidas de autointoxicação por pesticidas, com 31,4\% ( $n=21$ ), conforme se visualiza na Tabela III. 


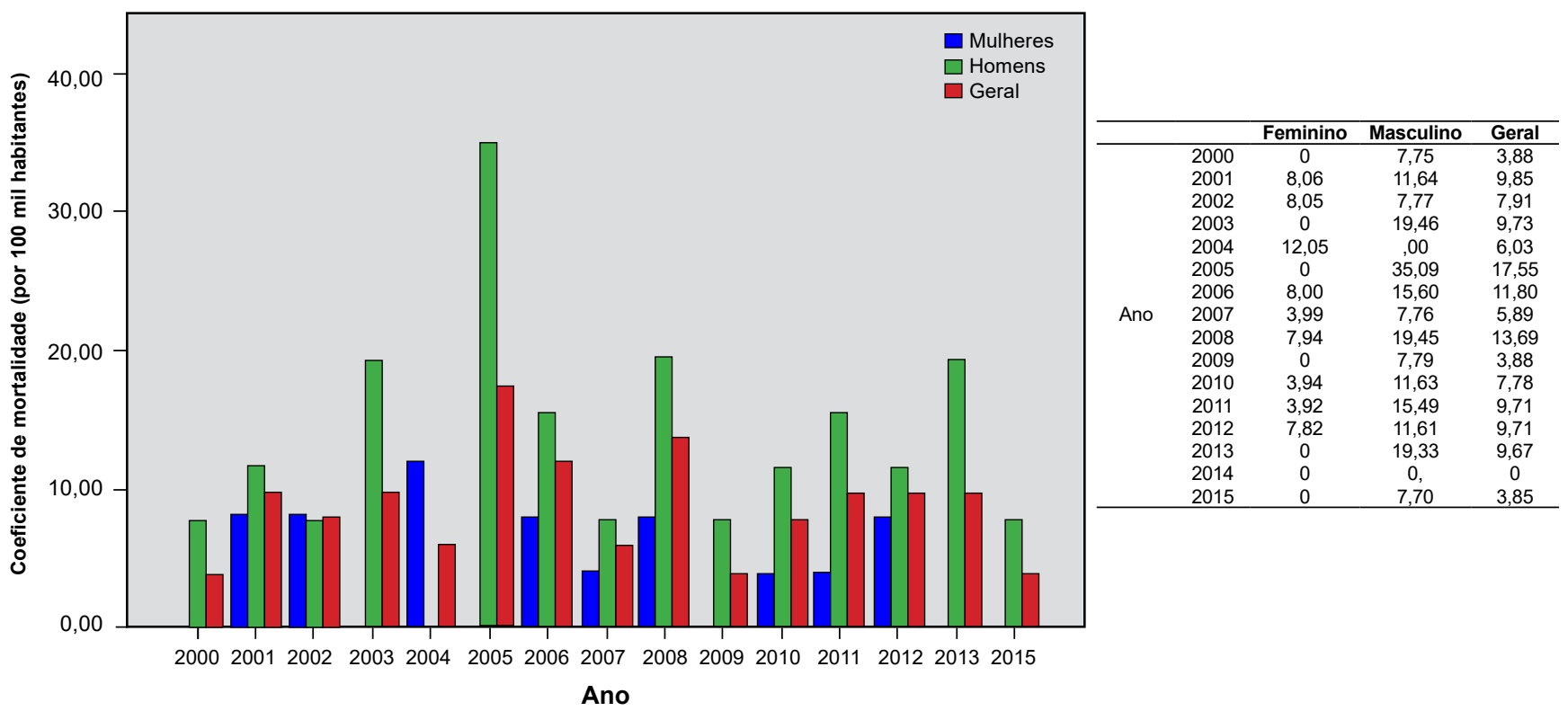

Figura 1 - Coeficiente de mortalidade por suicídio (em 100.000 habitantes), segundo gênero e população geral. Morada Nova, Ceará, Brasil (2000-2015).

Fonte: SIM: Sistema de Informação sobre Mortalidade; DATASUS (2020)

Tabela I - Caracterização sociodemográfica dos casos de óbito por suicídio em Morada Nova, Ceará, Brasil (2000-2015).

\begin{tabular}{|c|c|c|c|}
\hline \multicolumn{2}{|c|}{ Caracterização sociodemográfica } & \multirow{2}{*}{$\begin{array}{c}\mathbf{n} \\
51\end{array}$} & \multirow{2}{*}{$\frac{\%}{76,1 \%}$} \\
\hline \multirow{3}{*}{ Gênero } & Masculino & & \\
\hline & Feminino & 16 & $23,9 \%$ \\
\hline & Total & 67 & $100 \%$ \\
\hline \multirow{8}{*}{ Faixa etária (em anos) } & $10-19$ & 5 & $7,5 \%$ \\
\hline & $20-29$ & 14 & $20,9 \%$ \\
\hline & $30-39$ & 10 & $14,9 \%$ \\
\hline & $40-49$ & 22 & $32,8 \%$ \\
\hline & $50-59$ & 8 & $11,9 \%$ \\
\hline & $60-69$ & 7 & $10,4 \%$ \\
\hline & $70-79$ & 1 & $1,5 \%$ \\
\hline & Total & 67 & $100 \%$ \\
\hline \multirow{5}{*}{ Estado civil } & Solteiro(a) & 35 & $53,0 \%$ \\
\hline & Casado(a) & 25 & $37,9 \%$ \\
\hline & Viúvo(a) & 3 & $4,5 \%$ \\
\hline & Separado(a) judicialmente & 3 & $4,5 \%$ \\
\hline & Total & 66 & $100 \%$ \\
\hline \multirow{5}{*}{ Raça/cor } & Branca & 16 & $28,0 \%$ \\
\hline & Preta & 1 & $1,8 \%$ \\
\hline & Parda & 39 & $68,4 \%$ \\
\hline & Indígena & 1 & $1,8 \%$ \\
\hline & Total & 57 & $100 \%$ \\
\hline \multirow{7}{*}{ Ocupação } & Agropecuária, florestais e da pesca & 43 & $69,3 \%$ \\
\hline & Serviços de reparação e manutenção & 7 & $11,3 \%$ \\
\hline & Membros do poder público e dirigentes de organizações & 4 & $6,5 \%$ \\
\hline & Forças armadas, policiais e bombeiros & 3 & $4,8 \%$ \\
\hline & Produção de bens e serviços industriais & 3 & $4,8 \%$ \\
\hline & Outros & 2 & $3,3 \%$ \\
\hline & Total & 62 & $100 \%$ \\
\hline
\end{tabular}

Fonte: SIM: Sistema de Informação sobre Mortalidade; DATASUS (2020) 
Tabela II - Coeficientes de mortalidade por suicídio, segundo faixa etária, em Morada Nova, Ceará, Brasil (2000-2015).

\begin{tabular}{|c|c|c|c|c|c|c|c|c|}
\hline Faixa etária & & $\begin{array}{l}10-19 \\
\text { anos }\end{array}$ & $\begin{array}{c}20-29 \\
\text { anos }\end{array}$ & $\begin{array}{c}30-39 \\
\text { anos }\end{array}$ & $\begin{array}{r}40-49 \\
\text { anos }\end{array}$ & $\begin{array}{c}50-59 \\
\text { anos }\end{array}$ & $\begin{array}{c}60-69 \\
\text { anos }\end{array}$ & $\begin{array}{l}70-79 \\
\text { anos }\end{array}$ \\
\hline & 2000 & - & - & 11,34 & 16,62 & - & - & - \\
\hline & 2001 & 6,63 & 28,40 & - & - & 21,43 & - & - \\
\hline & 2002 & - & 9,37 & 11,30 & 31,58 & - & - & - \\
\hline & 2003 & 7,03 & 9,28 & 11,33 & 30,62 & - & - & - \\
\hline & 2004 & - & - & - & 29,70 & 20,43 & - & - \\
\hline & 2005 & - & 18,34 & 22,81 & 28,89 & 20,14 & 57,50 & - \\
\hline & 2006 & - & 9,06 & - & 42,16 & 19,85 & 28,28 & - \\
\hline & 2007 & 7,81 & - & - & 13,69 & 19,61 & - & - \\
\hline \multirow[t]{9}{*}{ Ano } & 2008 & 7,96 & 26,89 & - & 13,36 & 19,38 & 27,14 & - \\
\hline & 2009 & - & - & 11,40 & 13,10 & - & - & - \\
\hline & 2010 & - & 9,18 & - & 25,79 & - & 25,77 & - \\
\hline & 2011 & - & 9,23 & 11,21 & 25,43 & - & 25,18 & - \\
\hline & 2012 & - & - & 33,27 & 12,57 & 17,46 & - & - \\
\hline & 2013 & - & 9,42 & - & 24,95 & 16,82 & - & 41,74 \\
\hline & 2014 & - & - & - & - & - & - & - \\
\hline & 2015 & 9,03 & - & - & - & - & 22,79 & - \\
\hline & Total & 2,56 & 8,61 & 7,51 & 20,57 & 10,34 & 12,44 & 2,78 \\
\hline
\end{tabular}

Fonte: SIM: Sistema de Informação sobre Mortalidade; DATASUS (2020)

Tabela III - Distribuição de métodos utilizados no suicídio de acordo com as categorias da CID-10*, Morada Nova, Ceará, Brasil (2000-2015).

\begin{tabular}{lrr}
\hline Distribuição de métodos utilizados no suicídio & $\mathbf{n}$ & \% \\
\hline Enforcamento, estrangulamento e sufocação (X70) & 33 & 49,2 \\
Pesticidas e produtos químicos (X68-X69) & 21 & 31,3 \\
Medicamentos, substâncias biológicas e não especificadas (X60-X64) & 3 & 4,5 \\
Arma branca e objetos contundentes (X78-X79) & 2 & 3,0 \\
Fumaça, pelo fogo e por chamas (X75-X77) & 2 & 3,0 \\
Meios não especificados (X84) & 6 & 9,0 \\
Total & 67 & 100,0 \\
\hline
\end{tabular}

Fonte: SIM: Sistema de Informação sobre Mortalidade; DATASUS (2020); *CID-10: Classificação Estatística Internacional de Doenças e Problemas Relacionados à Saúde

\section{DISCUSSÃO}

A cidade de Morada Nova apresentou coeficiente de mortalidade por suicídio (8,77/100 mil habitantes) superior ao coeficiente observado no estado do Ceará $(5,1 / 100$ mil habitantes) e ao coeficiente nacional $(5,5 / 100$ mil habitantes) ${ }^{(12,22,23)}$. No ano de 2005 , houve em Morada Nova um coeficiente de 17,8 óbitos por suicídio/100 mil habitantes, aproximadamente o triplo dos coeficientes estadual e nacional. Os casos de suicídio em zonas rurais apresentam crescimento significativo ${ }^{(24,25)}$. Um estudo brasileiro evidenciou associação com baixa renda, dificuldade de acesso aos serviços de saúde mental e redução na prescrição de medicamentos antidepressivos ${ }^{(26)}$.

A estratificação dos coeficientes de mortalidade por suicídio é considerada baixa quando o coeficiente é menor que 5 óbitos $/ 100$ mil habitantes, médio entre 5 e menos de 15 óbitos $/ 100$ mil habitantes, alto entre 15 e menos de 30 óbitos/100 mil habitantes, e muito alto quando há 30 ou mais óbitos/100 mil habitantes ${ }^{(27)}$. Conforme o critério 
citado, a mortalidade por suicídio em Morada Nova é considerada como média para a população total, porém, para o grupo na faixa etária dos 40-49 anos, apresenta nível alto.

Acerca da epidemiologia entre os gêneros, a relação de mortalidade entre homem/mulher tem sido constante nas séries históricas de diferentes estudos populacionais, sendo estimado na razão $3: 1^{(5,11,28)}$, que corrobora com os achados locais, correspondentes à proporção de 3,18:1. A associação do sexo com o método utilizado para tentar ou cometer o suicídio é evidente em levantamentos nacionais e estrangeiros ${ }^{(4,6,11)}$.

Os homens optam mais por métodos com maior letalidade, como enforcamento e uso de arma de fogo, do que as mulheres. A menor ocorrência de suicídio entre as mulheres têm sido atribuída à baixa prevalência do uso abusivo de substâncias e à presença de maior engajamento espiritual ${ }^{(4,23)}$. Além disso, as mulheres reconhecem precocemente sinais de risco para comportamento suicida e buscam com maior frequência as redes de saúde, especialmente durante episódios agudos de transtornos psiquiátricos ${ }^{(4)}$, enquanto os homens apresentam características e comportamentos mais propensos ao suicídio, incluindo impulsividade ${ }^{(8,23)}$.

Em Morada Nova, os maiores coeficientes de mortalidade, segundo o grupo etário, ocorrem na população com 40-49 anos, porém surge elevação em idosos de 60 aos 69 anos. O aumento da incidência de suicídio na população senil é evidenciado em outros estudos ${ }^{(0,23,29)}$. Um estudo que avaliou a mortalidade por suicídio em idosos na Bahia observou incremento de $206,3 \%$ no coeficiente de mortalidade, que passou de 2,2/100 mil habitantes em 1996 para $6,8 / 100$ mil habitantes em 2013, com incremento anual de $11 \%^{(29)}$.

O estado civil solteiro(a), predominante entre a população avaliada na cidade cearense, é reconhecidamente um dos fatores sociodemográficos com franca relação ao comportamento suicida, principalmente quando associado ao isolamento social. A situação conjugal majoritária apresentou frequência semelhante com estudo realizado com a população do Ceará, com frequência estimada entre $53-58 \%^{(22)}$.

Os trabalhadores ligados à ocupação de serviços agropecuários apresentaram maior frequência relativa de mortalidade por suicídio no município, com representação de $64,2 \%$ da amostra populacional. O suicídio tem sido relatado como preocupante problema em populações rurais, inclusive entre trabalhadores agrícolas, em diferentes regiões do mundo, como Brasil, Índia e Espanha ${ }^{(24,30,31)}$.

Os resultados de um estudo brasileiro, realizado em um município do Rio de Janeiro com uso elevado de pesticidas, apontou que a classe agropecuária apresentou razão de chance para óbito por suicídio com variação entre 4,5 a 9,4 em comparação à população geral( ${ }^{(25)}$. Na Índia, em 2015, cerca de 20.000 habitantes faleceram em consequência da autointoxicação por pesticidas ${ }^{(31)}$. Em 2011, após regulação e banimento do inseticida Endosulfan, houve pequena, porém relevante, diminuição das taxas gerais de suicídio e maior declínio nos coeficientes de suicídio ocasionados pelos pesticidas ${ }^{(31)}$.

$\mathrm{Na}$ Espanha, as taxas de suicídio reproduziram elevada prevalência em regiões com predomínio de atividade agrícola e com uso intenso de pesticidas ${ }^{(24)}$. A mortalidade elevada de agricultores por suicídio estaria refletindo as precárias condições de sobrevivência, as dificuldades econômicas e/ou a exposição intensa aos agrotóxicos/pesticidas ${ }^{(1,32,33)}$.

Os agrotóxicos podem ser absorvidos diretamente pelas mucosas, pelo trato gastrointestinal e pelas vias respiratórias. A exposição crônica aos agrotóxicos, em especial aos organofosforados, pode causar alterações no nível do sistema neuroendócrino. Assim, a estimulação excessiva exercida pelos agrotóxicos em receptores nervosos pode causar variações nos níveis de serotonina. Essas modificações na concentração de serotonina podem estar relacionadas com a etiologia de diversos transtornos psiquiátricos com desencadeamento de comportamento suicida ${ }^{(6)}$.

A implementação de regulamentos e estratégias de restrição aos meios de cometer suicídio, como o controle do uso de agrotóxicos/pesticidas e de armas de fogo por meio de pactos e regulamentações, pode reduzir a incidência do suicídio, sendo recomendadas como instrumentos de prevenção universal ${ }^{(23,34)}$. Vale salientar que, diante da rigidez da regulamentação de acesso aos outros meios, a maior acessibilidade e o grau de letalidade inerente ao enforcamento podem explicar o aumento do número de suicídios por esse meio(4).

A análise da tabulação dos dados sobre suicídio na cidade de Morada Nova, com base no SIM, demonstrou que, apesar da melhoria na qualidade das informações no registro das declarações de óbito, não houve registro no campo "escolaridade" na população afligida pelo suicídio. Esse dado revela a persistência de falhas na qualidade do preenchimento das declarações de óbito, apontando a necessidade de aprimoramento da qualidade dos registros e de obtenção de informações referentes às circunstâncias dos óbitos ${ }^{(23,28)}$. A falta de registros de escolaridade configurou-se como limitação, também presente em outro levantamento, que utilizou dados da população do estado do Ceará(2).

A subnotificação denota-se como outra limitação. A mortalidade por suicídio no Brasil pode ter números mais expressivos, tendo em vista a subnotificação decorrente do estigma social e de motivações socioculturais, que favorecem a omissão de casos e acarretam prejuízo no reconhecimento do panorama real da magnitude do suicídio em todo o 
território. Outro fator relevante para a subnotificação é a dificuldade dos serviços para identificar a intencionalidade das causas externas de morte. Causas como afogamento, intoxicação acidental, acidente automobilístico intencional e morte por causa indefinida camuflam considerável proporção de casos de suicídio ${ }^{(2,4,8,23)}$ utilizando os óbitos por suicídio analisados por regiões no período de 1996 a 2015 contidos no Sistema de Informações sobre Mortalidade. Além disso, observa-se evidente lacuna no panorama epidemiológico sobre os estudos do comportamento suicida em municípios de pequeno e médio porte ${ }^{(26)}$. Logo, este estudo visa a contribuir com a disseminação de conhecimento nessa perspectiva e, também, apontar para a necessidade de novos estudos na área.

\section{CONCLUSÃO}

Os dados de mortalidade por suicídio no município investigado encontram-se em consonância com as estatísticas globais, mas acima das taxas estaduais e nacionais de suicídio. O perfil das pessoas que cometeram suicídio é composto predominantemente por homens, de raça/cor parda, solteiros e que exercem atividade agrícola como ocupação.

\section{CONFLITOS DE INTERESSE}

Os autores declaram que não há conflitos de interesses.

\section{CONTRIBUIÇÕES}

Sérgio André de Souza Júnior contribuiu com a elaboração e delineamento do estudo; a aquisição, análise e interpretação de dados; e a redação e revisão do manuscrito. Cássia Ferreira Rodrigues contribuiu com a redação e a revisão do manuscrito.

\section{REFERÊNCIAS}

1. World Health Organization. National suicide prevention strategies: progress, examples and indicators [Internet]. Geneva: World Health Organization; 2018 [acesso em 2020 Jun 26]. Disponível em: https://apps. who.int/iris/bitstream/handle/10665/279765/9789241515016-eng.pdf?ua=1

2. Teixeira SMO, Souza LEC, Viana LMM. O suicídio como questão de saúde pública. Rev Bras Promoç Saúde. 2018;31(3):1-3.

3. World Health Organization. Suicide in the world: global health estimates [Internet]. Geneva: World Health Organization; 2019 [acesso em 2020 Jun 26]. Disponível em: https://apps.who.int/iris/bitstream/ handle/10665/326948/WHO-MSD-MER-19.3-eng. pdf? sequence=1\&isAllowed=y

4. Botega NJ. Comportamento suicida: epidemiologia. Psicol USP. 2014;25(3):231-6.

5. Galvão PVM, Silva HRS, Silva CMFP. Temporal distribution of suicide mortality: a systematic review. J Affect Disord. 2018;228:132-42.

6. Sinyor M, Tse R, Pirkis J. Global trends in suicide epidemiology. Curr Opin Psychiatry. 2017;30(1):1-6.

7. Mann JJ, Apter A, Bertolote J, Beautrais A, Currier D, Haas A, et al. Suicide Prevention Strategies. JAMA. 2005;294(16):2064-74.

8. D'Eça A Jr, Rodrigues LS, Meneses EP Filho, Costa LDLN, Rêgo AS, Costa LC, et al. Mortalidade por suicídio na população brasileira, 1996-2015: qual é a tendência predominante? Cad Saúde Coletiva. 2019;27(1):20-4.

9. Rodrigues CD, Souza DS, Rodrigues HM, Konstantyner TCRO. Trends in suicide rates in Brazil from 1997 to 2015. Brazilian J Psychiatry. 2019;41(5):380-88.

10. Ministério da Saúde (BR), Secretaria de Vigilância em Saúde, Secretaria de Atenção à Saúde. Política Nacional de Promoção da Saúde: PNPS: Anexo I da Portaria de Consolidação $n^{\circ} 2$, de 28 de setembro de 2017, que consolida as normas sobre as políticas nacionais de saúde do SUS [Internet]. Brasília: Ministério da Saúde; 2018 [acesso em 2020 Jun 26]. Disponível em: http://bvsms.saude.gov.br/bvs/publicacoes/politica_ nacional_promocao_saude.pdf

11. Martini M, Fonseca RC, Sousa MH, Azambuja CF, Cardoso TA, Kunz M, et al. Age and sex trends for suicide in Brazil between 2000 and 2016. Soc Psychiatry Psychiatr Epidemiol. 2019;54(7):857-60. 
12. Martins DF Jr, Felzemburgh RM, Dias AB, Caribé AC, Bezerra-Filho S, Miranda-Scippa Â. Suicide attempts in Brazil, 1998-2014: an ecological study. BMC Public Health. 2016;16(1):4-11.

13. Dantas AP, Azevedo UN, Nunes AD, Amador AE, Marques MV, Barbosa IR. Analysis of suicide mortality in Brazil: spatial distribution and socioeconomic context. Rev Bras Psiquiatr. 2018;40(1):12-8.

14. World Health Organization. Preventing suicide: a global imperative [Internet]. Geneva: World Health Organization; 2014 [acesso em 2019 Nov 18]. Disponível em: https://apps.who.int/iris/bitstream/ handle/10665/131056/9789241564779_eng.pdf?sequence=1

15. Jacob KS. Reducing suicide rates: need for public health and population interventions. Indian J Psychol Med. 2016;38(6):510-13.

16. Machado MFS, Leite CKS, Bando DH. Políticas públicas de prevenção do suicídio no Brasil: uma revisão sistemática. Rev Gest Polít Públicas. 2014;4(2):334-56.

17. Brasil. Lei n. 13.819, de 26 de abril de 2019. Institui a política nacional de prevenção da automutilação e do suicídio [Internet]. Diário Oficial da União: secão 1, Brasília, DF, n. 81, 2019 [acesso em 2020 Jan 13]. Disponível em: http://www.planalto.gov.br/ccivil_03/_ato2019-2022/2019/lei/L13819.htm

18. Instituto Brasileiro de Geografia e Estatística. Base de informações do censo demográfico 2010: resultados do Universo por setor censitário [Internet]. Rio de Janeiro: IBGE; 2011 [acesso em 2020 Fev 25]. Disponível em: ftp://ftp.ibge.gov.br/Censos/Censo_Demografico_2010/Resultados_do_Universo/Agregados_por_ Setores_Censitarios/CE_20171016.zip

19. Instituto Brasileiro de Geografia e Estatística. Estimativas da população residente com data de referência $1^{\circ}$ de julho de 2019 [Internet]. Rio de Janeiro: IBGE; 2019 [acesso em 2020 Fev 25]. Disponível em: https:// biblioteca.ibge.gov.br/visualizacao/livros/liv101662.pdf

20. Organização Mundial da Saúde. CID-10 Classificação Estatística Internacional de Doenças e Problemas Relacionados à Saúde. São Paulo: Universidade de São Paulo; 1997.

21. Meirelles RC Jr, Castro JO, Faria LR, Silva CLA, Alves WA. Notificações de óbitos por causas externas e violência contra idosos: uma realidade velada. Rev Bras Promoç Saúde. 2019;32:1-12.

22. Oliveira MIV, Bezerra JG Filho, Feitosa RFG. Estudo Epidemiológico da Mortalidade por suicídio no Estado do Ceará no período 1997-2007. Rev Baiana Saúde Pública. 2012;36(1):159-73.

23. Ministério da Saúde (BR). Perfil epidemiológico das tentativas e óbitos por suicídio no Brasil e a rede de atenção à saúde: boletim epidemiológico. Brasília: Ministério da Saúde; 2017.

24. Freire $C$, Koifman S. Pesticides, depression and suicide: a systematic review of the epidemiological evidence. Int J Hyg Environ Health. 2013;216(4):445-60.

25. Meyer A, Koifman S, Koifman RJ, Moreira JC, Rezende JC, Abreu-Villaça Y. Mood disorders hospitalizations, suicide attempts, and suicide mortality among agricultural workers and residents in an area with intensive use of pesticides in Brazil. J Toxicol Environ Health. 2010;73(14):866-77.

26. Marín-León L, Oliveira HB, Botega NJ. Suicide in Brazil, 2004-2010: the importance of small counties. Pan Am J Public Heal. 2012;32(5):351-59.

27. Diekstra RFW, Gulbinat W. The epidemiology of suicidal behaviour: a review of three continents. World Heal Stat Q. 1993;46(1):52-68.

28. Machado DB, Santos DN. Suicídio no Brasil, de 2000 a 2012. J Bras Psiquiatr. 2015;64(1):45-54.

29. Carmo ÉA, Santos PHS, Ribeiro BS, Soares CJ, Santana MLADA, Bomfim EDS, et al. Características sociodemográficas e série temporal da mortalidade por suicídio em idosos no estado da Bahia, 1996-2013. Epidemiol Serv Saude. 2018;27(1):1-8.

30. Mew EJ, Padmanathan P, Konradsen F, Eddleston M, Chang SS, Phillips MR, et al. The global burden of fatal self-poisoning with pesticides 2006-15: systematic review. J Affect Disord. 2017;219:93-104.

31. Bonvoisin T, Utyasheva L, Knipe D, Gunnell D, Eddleston M. Suicide by pesticide poisoning in India: a review of pesticide regulations and their impact on suicide trends. BMC Public Health. 2020;20(1):1-16. 
32. Krawczyk N, Meyer A, Fonseca M, Lima J. Suicide mortality among agricultural workers in a region with intensive tobacco farming and use of pesticides in Brazil. J Occup Environ Med. 2014;56(9):993-1000.

33. World Health Organization. Preventing suicide: a resource for pesticide registrars and regulators [Internet]. Geneva: World Health Organization; 2019 [acesso em 2020 Jun 26]. Disponível em: https://apps.who.int/iris/ rest/bitstreams/1244790/retrieve

34. Zalsman G, Hawton K, Wasserman D, van Heeringen K, Arensman E, Sarchiapone M, et al. Suicide prevention strategies revisited: 10-year systematic review. Lancet Psychiatry. 2016;3(7):646-59.

Endereço para correspondência:

Sérgio André de Souza Júnior

Curso de Medicina da Universidade de Fortaleza

Avenida Washington Soares, 1321

Bairro: Edson Queiroz

Cep: 60811-905 - Fortaleza - CE - Brasil

E-mail: sergioandre@edu.unifor.br

Como citar: Souza SA Jr, Rodrigues CF. Mortalidade por suicídio: realidade de uma cidade no interior do nordeste brasileiro. Rev Bras Promoç Saúde. 2020;33:10813. 